\title{
The effect of artichoke leaf extract supplementation on lipid profile of chronic kidney disease patients; a double- blind, randomized clinical trial
}

\author{
Seyed Mansour Gatmiri ${ }^{1+}{ }^{\circledR}$, Ebrahim Khadem $^{2 \dagger}{ }^{\circledR}$, Toktam Fakhrian $^{3}$, Mohammad Kamalinejad ${ }^{4}$, Hamed $^{\circ}$ \\ Hosseini $^{5}$, Fereshteh Ghorat $^{6}$, Azam Alamdari ${ }^{*}{ }^{*}$, Neda Naderi $^{1^{*}(\mathbb{0}}$ \\ ${ }^{1}$ Nephrology Research Center, Center of Excellence in Nephrology, Tehran University of Medical Sciences, Tehran, Iran \\ ${ }^{2}$ Department of Persian Medicine, School of Persian Medicine, Tehran University of Medical Sciences, Tehran, Iran \\ ${ }^{3}$ School of Medicine, Tehran University of Medical Sciences, Tehran, Iran \\ ${ }^{4}$ School of Pharmacy, Shahid Beheshti University of Medical Sciences, Tehran, Iran \\ ${ }^{5}$ Center for Research and Training in Skin Diseases and Leprosy, Tehran University of Medical Sciences \\ ${ }^{6}$ School of Medicine, Sabzevar University of Medical Sciences, Sabzevar, Iran \\ ${ }^{\dagger}$ These authors contributed equally
}

\section{A R T I C L E I N F O}

Article Type:

Original

\section{Article History:}

Received: 3 April 2019

Accepted: 19 June 2019

Published online: 11 July 2019

Keywords:

Artichoke

Chronic kidney disease

Dietary supplement

Hypercholesterolemia

Cholesterol

Hyperlipidemia

Cynara scolymus

\begin{abstract}
A B S T RA C T
Introduction: Hyperlipidemia is considered a prominent risk factor for coronary heart disease. Coronary heart disease is the most common cause of mortality and morbidity in chronic kidney disease (CKD) patients.

Objectives: The aim of this study was to evaluate the effects of artichoke leaf extract (ALE) supplementation on the lipid profile of CKD patients.

Patients and Methods: A randomized, double-blind, placebo-controlled clinical trial was conducted in $38 \mathrm{CKD}$ subjects (GFR $<60$ and $>15 \mathrm{~mL} / \mathrm{min} / 1.73 \mathrm{~m}^{2}$ ) with hypercholesterolemia (total cholesterol $\geq 200 \mathrm{mg} / \mathrm{dL}$ or low-density lipoprotein cholesterol [LDL-C] $\geq 110 \mathrm{mg} / \mathrm{dL}$ ). The intervention group received an artichoke capsule $(320 \mathrm{mg}$ ) and an indistinguishable placebo was given to the control group twice daily for 6 weeks. Lipid profile and appetite were assessed at week 8 and the results were compared with the baseline data.

Results: ALE supplementation was correlated with a statistically significant decrease in mean total cholesterol $(P=0.028)$ and LDL-C $(P=0.005)$ compared to the control group. The two groups did not show a significant difference in high-density lipoprotein-cholesterol $(P=0.071)$ and triglyceride levels $(P=0.22)$. There was a statistically significant difference in appetite between the two groups $(P=0.016)$.

Conclusion: This randomized controlled trial demonstrated that consumption of ALE supplement may improve appetite and lipid profile in CKD patients.

Trial Registration: This RCT has been approved by Iranian Registry of Clinical Trials identifier: IRCT2016021622689N3; http://en.irct.ir/trial/19516, Ethics committee reference number; Ir.tums.rec.1394.1531).
\end{abstract}

\section{Implication for health policy/practice/research/medical education}

The aim of this study was to evaluate the effects of ALE supplement on the lipid profile of CKD patients. This randomized controlled trial demonstrated that consumption of ALE supplement may improve lipid profile and appetite in these patients. Please cite this paper as: Gatmiri SM, Khadem E, Fakhrian T, Kamalinejad M, Hosseini H, Ghorat F, et al. The effect of artichoke leaf extract supplementation on lipid profile of chronic kidney disease patients; a double-blind, randomized clinical trial. J Renal Inj Prev. 2019; 8(3): 225-229. DOI: 10.15171/jrip.2019.42.

\section{Introduction}

Chronic kidney disease (CKD) is a worldwide health issue with a growing incidence and prevalence particularly due to population aging and increasing frequency of comorbidities (1). CKD is associated with a higher cardiovascular mortality. Cardiovascular disease (CVD) is the main cause of death and morbidity in these patients (2) and cardiovascular mortality is much higher in CKD stages 4-5 compared to the general population (3).

Although high serum concentrations of triglyceride (TG), total cholesterol and low-density lipoprotein cholesterol (LDL-C) and low serum levels of high-density 
lipoprotein cholesterol (HDL-C) are known risk factors for the CVD development in the general population, an inverse association between non-HDL-C which mainly consist of TG rich lipoproteins and mortality in end-stage renal disease (ESRD) patients transitioning to dialysis has been described (4).

CKD patients who do not have nephrotic syndrome and do not need renal replacement therapy, usually have low HDL-C, high TG and normal LDL-C which is smaller, denser and more atherogenic (5). Dyslipidemia which is a common complication of CKD has a special role in the development of atherosclerosis and is the principal modifiable risk factor for cardiovascular disease (6). It also has been a target for pharmacological therapeutic agents and natural lipid lowering compounds in primary and secondary prevention (7).

Cynara scolymus (Artichoke) is an herbaceous perennial plant which its' leaves is widely used as hepato-protective, diuretic, anti-oxidative and cholesterol biosynthesis inhibitor (8). A few mechanisms of action for lipid lowering effect of artichoke leaf extract (ALE) have been considered. Interaction with 3-hydroxy-3-methylglutaryl coenzyme A (HMG-CoA) reductase, liver sterol regulatory elementbinding proteins and acetyl-CoA C-acetyltransferase (ACAT) by Luteolin are proposed mechanisms (9).

\section{Objectives}

In this randomized, double-blind, placebo-controlled clinical trial we evaluated the effect of 6 weeks ALE supplementation on the lipid profile of our CKD patients.

\section{Patients and Methods \\ Study design}

This double-blind, placebo controlled, randomized clinical trial was conducted in CKD patients who were referred to the nephrology clinic of Imam-Khomeini hospital in Tehran, Iran from December 2017 to July 2018. The participants were randomly assigned into two groups ( $n=20$ patients per group) through block randomization using quadruple blocks. Blocks were randomly selected to make the randomization list. The drug and placebo were administered to the patients in similar packages and the same dose. The researcher and patients were blind to nature of the intervention (Figure 1).

\section{Participants}

Totally $64 \mathrm{CKD}$ patients were consequently enrolled in the study (Figure 1). Forty participants met the inclusion criteria consisting of estimated glomerular filtration rate (eGFR) between $15-59 \mathrm{~mL} / \mathrm{min} / 1.73 \mathrm{~m}^{2}$ (stages $3 \mathrm{a}, 3 \mathrm{~b}$ and 4 ), LDL-C level $\geq 110 \mathrm{mg} / \mathrm{dL}$ or total cholesterol level $\geq 200 \mathrm{mg} / \mathrm{dL}$, age between 18 and 70 years, body mass index (BMI) value between $18.5 \mathrm{~kg} / \mathrm{m}^{2}$ and $30 \mathrm{~kg} / \mathrm{m}^{2}$, positive history of low lipid diet and statin use for more than 4 months. Patients with severe chronic disease, type
2 diabetes, liver function impairment, history of biliary obstructive disorder or gallstone, history of dialysis or kidney transplantation, pregnancy or breastfeeding, known or potential allergy to the product under the study, participation in any other clinical trial at the same time or within the last 30 days, history of malignancy and drug abuse, were excluded.

\section{Intervention}

Patients were allocated to receive a capsule containing $320 \mathrm{mg}$ of ALE or an indistinguishable placebo capsule two times a day for 6 weeks. All participants were asked to continue their regular diet and medications. Anthropometrical parameters, such as body weight and BMI were collected both at the beginning and two weeks after the trial. Biochemical parameters have been obtained at weeks 0 and 8, after a 12-hour overnight fasting. Adherence to treatment/placebo, tolerability, acceptability and compliance were also assessed.

\section{Ethical issues}

The study was conducted in accordance with the Declaration of Helsinki. The protocol was approved by the Ethics Committee of Tehran University of Medical Sciences (Ir.tums.rec.1394.1531). Informed consent was obtained from all participants before inclusion in the study and they were informed of their right to withdraw from the study at any time. This study was also registered in Iranian Registry of Clinical Trials (IRCT) (\# IRCT2016021622689N3; http://en.irct.ir/trial/19516; registration date: 2016-0618). This study was part of Toktam Fakhrian dissertation, granted by Tehran University of Medical Sciences (Grant\# 94-3146-30407).

\section{Statistical analysis}

We used SPSS (SPSS Inc., Chicago, IL, USA) version 16.0 software for descriptive statistics. Parametric variables were described using mean and standard deviation and non-parametric data by using frequency. Parametric variables were evaluated by Student's $t$ test. Means were compared using analysis of variance (ANOVA). $P$ values less than 0.05 were accepted as statistically significant.

\section{Results}

In this clinical trial 20 patients were randomized to ALE supplement and 20 to placebo. Nineteen patients completed the 6-week intervention in each arm. Mean age of the participants was $50.24 \pm 11.63$ years $(n=38)$ and there was no statistically significant difference between comparison groups $(P=0.92)$. The subjects were predominantly women $(60.5 \%)$. Demographic and baseline laboratory characteristics of each group are presented in Table 1. There were not any significant differences in the baseline laboratory characteristics between the participants in each group. 


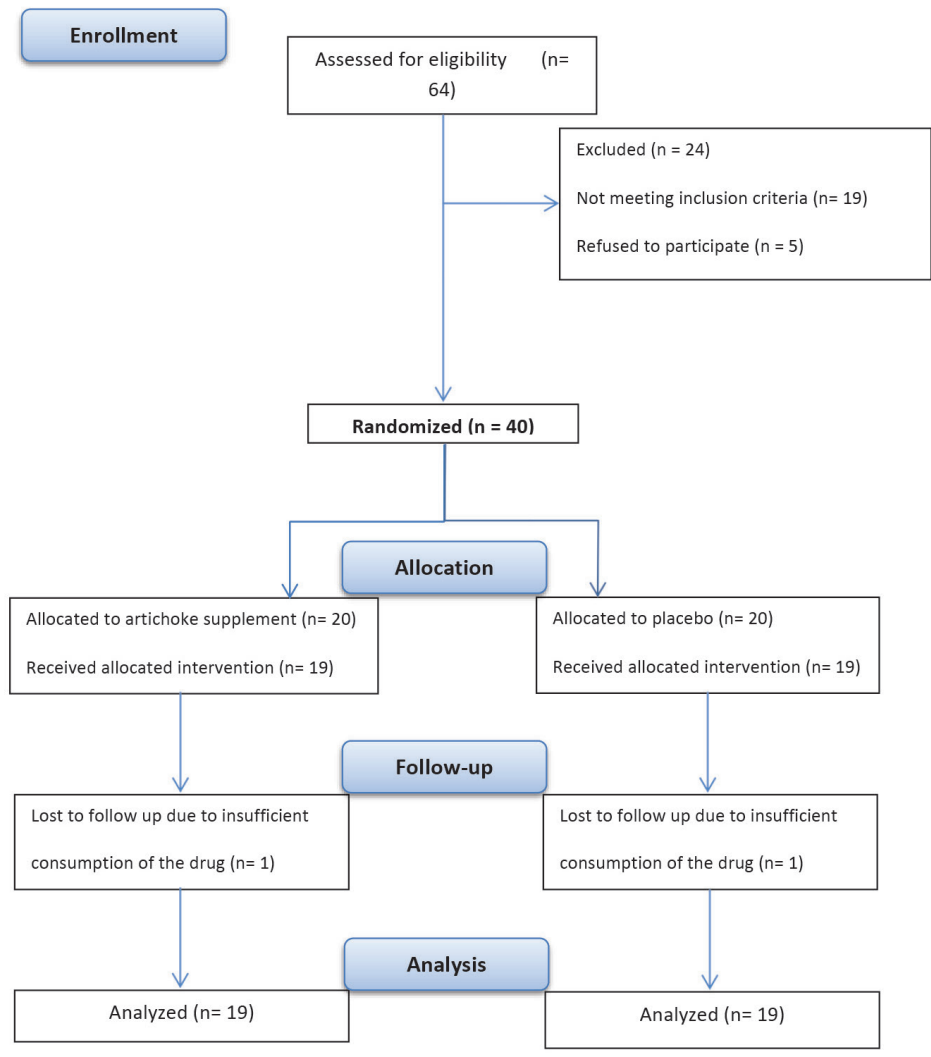

Figure 1. Flowchart of patient selection for the study.

At the end of the study, compliance with artichoke and placebo capsules were $97.6 \%$ and $95.8 \%$ of expected doses, respectively $(P=0.38)$. Mean total cholesterol in the supplement group was $202 \pm 8.18 \mathrm{mg} / \mathrm{dL}$ and in the placebo group was $228 \pm 8.18 \mathrm{mg} / \mathrm{dL}$, the mean difference was $-26.57 \pm 11.59 \mathrm{mg} / \mathrm{dL}$ that was statistically significant (CI: $-50.1--3.0, P=0.028$ ). Mean LDL-C in the ALE supplement group was $119 \pm 5.9 \mathrm{mg} / \mathrm{dL}$, in the placebo group was $144 \pm 5.9 \mathrm{mg} / \mathrm{dL}$ and the mean difference was $-25.09 \pm 8.45 \mathrm{mg} / \mathrm{dL}$ that was statistically significant (95\% CI -42.24- -7.93, $P=0.005)$. Mean HDL-C in ALE group was $44.53 \pm 0.8 \mathrm{mg} / \mathrm{dL}$ and in the other group was $42.25 \pm 0.8 \mathrm{mg} / \mathrm{dL}$ while, the mean difference was not statistically significant between the two groups (95\% CI -0.2-4.7, $P=0.071$ ). Comparing the LDL-C to HDL-C ratio, a statistically significant difference (mean difference $=-0.70 \pm 0.18)$ between two groups was detected $(95 \% \mathrm{CI}$ -1.05- $-0.34, P<0.001)$.

Mean serum TG level in the placebo group was $202.8 \pm 14.81 \mathrm{mg} / \mathrm{dL}$ and in the artichoke group was $176.4 \pm 14.81 \mathrm{mg} / \mathrm{dL}$ since, there was not a statistically significant difference between the groups (95\% CI -69.5616.82, $P=0.22$ ).

To compare appetite based on the participants answer to visual analogue scale (VAS), a statistically significant improvement in appetite in ALE supplement group was found $(P=0.016)$. There was no significant difference in blood glucose level between the two groups at the end of the study.

Discussion

In this randomized, double-blind placebo-controlled trial of $38 \mathrm{CKD}$ individuals (stages $3 \mathrm{a}-4$ ), with mild to moderate hypercholesterolemia, we found that a 6-week supplementation with ALE (320 mg, twice daily) was associated with a significant reduction in serum total cholesterol and LDL-C level, without any significant changes in serum triglycerides or HDL-C level.

Active components of ALE are flavonoids (luteolin and glucosides), caffeoylquinic acids (cynarin and chlorogenic acid), bitters (sesquiterpene lactones, including cynaropicrin), and caffeic acids $(10,11)$. it seems that chlorogenic acid and luteolin which are found in considerable amounts in artichoke, have an important role in lipid lowering mechanisms (12). Proposed mechanisms by which ALE may exert lipid-lowering effects are interaction of luteolin with HMG-CoA reductase, liver sterol regulatory element-binding proteins and ACAT, increasing cholesterol elimination through bile acid secretions and inhibition of LDL oxidation $(10,13)$.

The clinical impact of ALE has been evaluated in several studies proposing potential lipid-lowering effects of ALE in hypercholesteremic adults (10, 1316). Bundy et al evaluated the effect of $1280 \mathrm{mg}$ standardized ALE administered daily for 12 weeks in healthy hypercholesteremic adults in a randomized, 
Table 1. Distribution of demographic characteristics and laboratory data of the participants before the intervention

\begin{tabular}{|c|c|c|c|}
\hline Variable & ALE supplement & Placebo & $P$ value \\
\hline Age (y) & $50.42 \pm 11.77$ & $50.05 \pm 11.48$ & 0.92 \\
\hline Sex & & & 0.74 \\
\hline Male & 7 & 8 & \\
\hline Female & 12 & 11 & \\
\hline $\mathrm{BMI}\left(\mathrm{kg} / \mathrm{m}^{2}\right)$ & $27.31 \pm 2.56$ & $26.57 \pm 4.19$ & 0.51 \\
\hline LDL (mg/dL) & $159.9 \pm 29.5$ & $143.8 \pm 40.3$ & 0.16 \\
\hline $\mathrm{HDL}(\mathrm{mg} / \mathrm{dL})$ & $43.5 \pm 7.9$ & $42.9 \pm 9.0$ & 0.83 \\
\hline Total CHOL (mg/dL) & $241.6 \pm 35.8$ & $233.5 \pm 46.9$ & 0.55 \\
\hline TG (mg/dL) & $177.8 \pm 64.9$ & $235.9 \pm 150.7$ & 0.13 \\
\hline LDL /HDL & $3.8 \pm 0.8$ & $3.4 \pm 0.9$ & 0.22 \\
\hline BUN (mg/dL) & $15.6 \pm 6.0$ & $20.3 \pm 14.7$ & 0.21 \\
\hline $\mathrm{Cr}(\mathrm{mg} / \mathrm{dL})$ & $1.1 \pm 0.3$ & $1.4 \pm 0.9$ & 0.15 \\
\hline WBC (cells/mcl) & $7246.1 \pm 2351.4$ & $6254.3 \pm 1155.9$ & 0.23 \\
\hline $\mathrm{Hb}(\mathrm{g} / \mathrm{dL})$ & $13.7 \pm 2.2$ & $13.9 \pm 0.8$ & 0.79 \\
\hline PLT (cells/mcl) & $256000 \pm 70579$ & $264000 \pm 28589$ & 0.75 \\
\hline AST (IU/I) & $27.5 \pm 14.6$ & $23.0 \pm 11.7$ & 0.52 \\
\hline ALT (IU/I) & $36.6 \pm 22.1$ & $31.7 \pm 27.7$ & 0.68 \\
\hline Alkp (IU/I) & $226.6 \pm 66.7$ & $111.0 \pm 45.2$ & 0.05 \\
\hline FBS (mg/dL) & $135.8 \pm 75.0$ & $144.0 \pm 34.8$ & 0.28 \\
\hline $\mathrm{Fe}(\mathrm{mcg} / \mathrm{dL})$ & $68.5 \pm 24.7$ & $62.0 \pm 9.9$ & 0.42 \\
\hline $\operatorname{ESR}(\mathrm{mm} / \mathrm{h})$ & $15.7 \pm 12.0$ & $25.3 \pm 14.1$ & 0.42 \\
\hline TIBC (mg/dL) & $304.5 \pm 30.4$ & $369.5 \pm 133.6$ & 0.57 \\
\hline
\end{tabular}

double blind placebo controlled trial. Although they found a statistically significant difference between two groups in total cholesterol, there was no significant difference regarding TG, LDL-C and HDL-C (10). In another study in 2006, Nazni et al examined the effect of ALE on lipid profile in type 2 diabetic individuals. The results of this study indicated a significant reduction in total cholesterol, triglyceride and LDL-C levels and also a significant increase in HDL-C levels was seen. In addition, the beneficial effect of ALE on blood glucose level was observed in this controlled trial (17). In a randomized, double-blind, placebo-controlled clinical trial in 2013, Rondanelli et al considered the effects of ALE on lipid patterns in 92 overweight subjects affected by primary mild hypercholesterolemia. The results of this study indicated that not only ALE decreases serum total cholesterol and LDL-C, but also it is associated with a significant increase in HDL-C levels (14). Recently, a systematic review and meta-analysis was carried out on 9 RCTs (included 702 subjects) investigating the impact of ALE on plasma lipid levels. The authors found a significant decrease in serum total cholesterol, LDL-C, and triglyceride levels following ALE administration, however no significant alteration was observed in HDL-C levels. Moreover, a significant association between baseline LDL-C level and lipidlowering effect of ALE was observed (13).
It should be mentioned that most of the above studies have been performed in patients with normal renal function. Dyslipidemia is common in CKD patients and is associated with high cardiovascular morbidity and mortality (5). Individuals with non-dialysis-dependent CKD have specific feature of lipid profile that is distinct from the general population and is characterized by low serum HDL-C, high triglycerides and normal or even low total cholesterol and LDL-C levels. Of note, LDL-C is not usually elevated, LDL-C particles are smaller, denser and more atherogenic, while oxidized LDL-C which is highly atherogenic, is increased in this population $(5,18,19)$.

In CKD, dyslipidemia accelerates atherogenic processes and in turn increases cardiovascular disease. Additionally, it accelerates decline in kidney function due to lipid deposition in the kidneys. Therefore, it is logical to control dyslipidemia in patients with CKD. According to the KDIGO guidelines, statin or statin/ezetimibe is recommended in patients with CKD who fulfill treatment criteria (5). Artichoke is one of the safest herbal medicines and has not been associated with significant side effects in most of the subjects (11). To the best of our knowledge, this is the first study to demonstrate the impacts of artichoke on lipid profile of CKD patient and our results are in concordant with studies performing in patients with normal kidney function. In this study, no adverse events related to treatment were reported and even patients' compliance was good.

In addition, there was a significant difference in appetite, favoring those participants receiving ALE supplement. This was consistent with the reports of a review study by Wegener et al in 1999 showed that ALE improved gastrointestinal complaints such as nausea, abdominal pain, and loss of appetite in patients with hepatobiliary dysfunction. It was probably, flavonoids and caffeoylquinic that were mainly responsible for these actions(20).

\section{Conclusion}

In conclusion, this study demonstrated that consumption of ALE supplement may improve appetite and lipid profile in CKD patients.

\section{Limitations of the study}

The small number of participants is an important limitation of this study. In addition, antioxidant effects of artichoke were not assessed in these CKD patients. Hence, further prospective and long- term clinical studies are necessary to clarify whether artichoke has other beneficial effects in CKD patients similar to the general population.

\section{Authors' contribution}

SMG and EK joined in concept, design and editing. $\mathrm{NN}$ and AA contributed to manuscript preparation and editing. TF joined in data collection and data analysis. $\mathrm{MK}, \mathrm{HH}$ and FG participated in study design and data analysis. 


\section{Conflicts of interest}

All authors declare no conflicts of interest.

\section{Ethical considerations}

Ethical issues (including plagiarism, data fabrication, double publication) have been completely observed by the authors.

\section{Funding/Support}

This study was supported by Tehran University of Medical Sciences research council with code of 940314630407.

\section{References}

1. Collister D, Ferguson T, Komenda P, Tangri N. The patterns, risk factors, and prediction of progression in chronic kidney disease: a narrative review. Semin Nephrol. 2016;36:273-82. doi:10.1016/j.semnephrol.2016.05.004.

2. Whitman IR, Feldman HI, Deo R. CKD and sudden cardiac death: epidemiology, mechanisms, and therapeutic approaches. J Am Soc Nephrol. 2012;23:1929-39. doi: 10.1681/asn.2012010037.

3. Sarnak MJ, Levey AS, Schoolwerth AC, Coresh J, Culleton B, Hamm LL, et al. Kidney disease as a risk factor for development of cardiovascular disease: a statement from the American Heart Association Councils on Kidney in Cardiovascular Disease, High Blood Pressure Research, Clinical Cardiology, and Epidemiology and Prevention. Hypertension. 2003;42:1050-65. doi: 10.1161/01. HYP.0000102971.85504.7c.

4. Chang TI, Streja E, Ko GJ, Naderi N, Rhee CM, Kovesdy CP, et al. Inverse association between serum non-high-density lipoprotein cholesterol levels and mortality in patients undergoing incident hemodialysis. J Am Heart Assoc. 2018;7(12). doi: 10.1161/jaha.118.009096.

5. Mikolasevic I, Žutelija M, Mavrinac V, Orlic L. Dyslipidemia in patients with chronic kidney disease: Etiology and management. Int J Nephrol Renovasc Dis. 2017;10:35-45. doi: 10.2147/IJNRD.S101808.

6. Weiner DE, Sarnak MJ. Managing dyslipidemia in chronic kidney disease. J Gen Intern Med. 2004;19:1045-52. doi: 10.1111/j.1525-1497.2004.40049.x.

7. Cicero AF, Colletti A, Fogacci F, Bove M, Rosticci M, Borghi C. Effects of a combined nutraceutical on lipid pattern, glucose metabolism and inflammatory parameters in moderately hypercholesterolemic subjects: a doubleblind, cross-over, randomized clinical trial. High Blood Press Cardiovasc Prev. 2017;24:13-8.

8. Kraft K. Artichoke leaf extract - Recent findings reflecting effects on lipid metabolism, liver and gastrointestinal tracts. Phytomedicine. 1997;4:369-78. doi: 10.1016/s09447113(97)80049-9.
9. Gebhardt R. Inhibition of cholesterol biosynthesis in HepG2 cells by artichoke extracts is reinforced by glucosidase pretreatment. Phytother Res. 2002;16:368-72. doi: 10.1002/ ptr.960.

10. Bundy R, Walker AF, Middleton RW, Wallis C, Simpson HC. Artichoke leaf extract (Cynara scolymus) reduces plasma cholesterol in otherwise healthy hypercholesterolemic adults: a randomized, double blind placebo controlled trial. Phytomedicine. 2008;15:668-75. doi: 10.1016/j. phymed.2008.03.001.

11. Ben Salem M, Ben Abdallah Kolsi R, Dhouibi R, Ksouda K, Charfi S, Yaich M, et al. Protective effects of Cynara scolymus leaves extract on metabolic disorders and oxidative stress in alloxan-diabetic rats. BMC Complement Altern Med. 2017;17:017-1835.

12. Santos HO, Bueno AA, Mota JF. The effect of artichoke on lipid profile: A review of possible mechanisms of action. Pharmacol Res. 2018;137:170-8.

13. Sahebkar A, Pirro M, Banach M, Mikhailidis DP, Atkin SL, Cicero AFG. Lipid-lowering activity of artichoke extracts: A systematic review and meta-analysis. Crit Rev Food Sci Nutr. 2017;13:1-8.

14. Rondanelli M, Giacosa A, Opizzi A, Faliva MA, Sala P, Perna S, et al. Beneficial effects of artichoke leaf extract supplementation on increasing HDL-cholesterol in subjects with primary mild hypercholesterolaemia: a double-blind, randomized, placebo-controlled trial. Int J Food Sci Nutr. 2013;64:7-15.

15. Englisch W, Beckers C, Unkauf M, Ruepp M, Zinserling V. Efficacy of Artichoke dry extract in patients with hyperlipoproteinemia. Arzneimittelforschung. 2000;50:260-5. doi: 10.1055/s-0031-1300196.

16. Wider B, Pittler MH, Thompson-Coon J, Ernst E. Artichoke leaf extract for treating hypercholesterolaemia. Cochrane Database Syst Rev. 2013;28(3).

17. Nazni P, Poongodi Vijayakumar T, Alagianambi P, Amirthaveni M. Hypoglycemic and hypolipidemic effect of Cynara scolymus among selected type 2 diabetic individuals. Pakistan Journal of Nutrition. 2006;5:147-51. doi: $\quad 10.3923 /$ pjn.2006.147.151.

18. Bermudez-Lopez M, Arroyo D, Betriu A, Masana L, Fernandez E, Valdivielso JM. New perspectives on CKD-induced dyslipidemia. Expert Opin Ther Targets. 2017;21:967-76. doi: 10.1080/14728222.2017.1369961.

19. Kujawa-Szewieczek A, Wiecek A, Piecha G. The lipid story in chronic kidney disease: a long story with a happy end? Int Urol Nephrol. 2013;45:1273-87. doi: 10.1007/s11255012-0296-8.

20. Wegener T, Fintelmann V. [Pharmacological properties and therapeutic profile of artichoke (Cynara scolymus L.)]. Wien Med Wochenschr. 1999;149:241-7.

Copyright ( $\odot 2019$ The Author(s); Published by Nickan Research Institute. This is an open-access article distributed under the terms of the Creative Commons Attribution License (http://creativecommons.org/licenses/by/4.0), which permits unrestricted use, distribution, and reproduction in any medium, provided the original work is properly cited. 